\title{
Preface: Advances in Theoretical and Practical Combinatorial Optimization
}

\author{
Said Salhi ${ }^{1}$, Richard Eglese ${ }^{2}$, Eleni Hadjiconstantinou ${ }^{3}$
}

This issue consists of research papers dedicated, though not limited, to the International Symposium in Combinatorial Optimisation (CO2016), chaired by Said Salhi and held at the University of Kent, Canterbury, 1-3 September 2016. Of the many good papers it attracted, 35 were considered suitable for the next stage of review, resulting in 20 being accepted mostly after two rounds of reviews. We divided the issue into three parts, each covering a wider though well-defined area within combinatorial optimization (CO). These three parts, which are not completely distinct, are referred to for simplicity as Mathematically-Oriented (MO), Heuristically-Based (HB) and Practically-Focussed (PF).

MO contains seven papers. These include a paper on the integration of network flows in a coloring algorithm by Koster, Scheidweiler, and Tieves; one on a polynomial time algorithm for the minimum flow problem by Khodayifar, Raayatpanah, and Pardalos, and one on a formulation in multi-level capacitated facility location by Irawan and Jones. A.Salhi, Alsoufi, and Yang provide a mixed integer program combined with an evolutionary approach for seaside operations in container ports, while Zare, Borrero, Zeng and Prokopyev have contributed a note on new linearized formulations in bilevel linear integer problems. Fuzzy logic is explored for portfolio optimization by Liagkouras and Metaxiotis, and for supplier selection by Chang.

HB covers six papers. Azizi, using a genetic algorithm, explores hub and spoke networks under disruption. Knust and Xie produce a simulated annealing algorithm for nurse rostering, while Pei, Cheng, Liu, Pardalos, and Kong investigate Variable Neighbourhood Search (VNS) for single and parallel machine serial batching. A skewed VNS is adopted by Derbel, Jarbaoui, and Bhiri for the heterogeneous fleet vehicle routing problem, and an ascent-escent VNS for the detection of community (cluster) structures in a network is discussed by Džamić, Aloise, and Mladenović. The last paper in this category is by Brimberg, Mladenović,

Todosijević, and Urošević, who investigate the capacitated clustering problem using a variant of VNS.

PF has seven papers that treat inspiring implementations of $\mathrm{CO}$ for real-life applications. For example, in the area of scheduling, the use of spare parts in vessel maintenance is dealt with by Kian, Bektaş, and Ouelhadj, while shift scheduling for power station staff is explored by Shuib and Kamarudin. The avoidance of obstacles in off shore wind farms is optimized by Klein and Haugland, and the costing of a product within a supply chain is performed by de Matta. In the health area, the maximization of the expected number of kidney transplants is performed by Alvelos, Klimentova, and Viana; a preventive health care network is designed by Davari; and the layout of an operating theater is produced by Chraibi, Osman, and Kharraja. 
Finally, we are grateful to Professor E. Boros for trusting our academic judgment in acting as guest editors of the Annals of Operations Research special issue devoted, but not restricted, to CO2016. We are also thankful to the managing editor, Katie D'Agosta, for her patience and valuable support in preparing this special issue.

${ }^{1}$ Centre for Logistics and Heuristic Optimisation, Kent Business School, University of Kent, Canterbury, UK

${ }^{2}$ Lancaster University Management School, Lancaster, UK

${ }^{3}$ School of Business and Law, Frederick University Cyprus, Cyprus. 